Informe especial

\title{
Elaboración de corredores o canales endémicos mediante planillas de cálculo
}

\author{
Marcelo Bortman ${ }^{1}$
}

RESUMEN Las epidemias o brotes pueden ser definidos como un exceso en el número de casos de un problema de salud dado, en una población, un período y un lugar en particular. Sin embargo, determinar lo que constituye un exceso implica conocer lo que es normal o de esperar. La elaboración de canales o corredores endémicos permite definir los valores de casos esperados y de esta forma evidenciar de forma gráfica la aparición de un número mayor de casos. En el presente trabajo se describe una nueva metodología para la realización de estos canales endémicos, en la que se utilizan las planillas de cálculo Qpro y Excel, mediante la determinación de la media geométrica de las tasas históricas y su intervalo de confianza. Se presenta también un corredor endémico acumulativo que facilita la vigilancia de sucesos endémicos de baja incidencia.

Probablemente, la definición más corta y simple de epidemia la formuló Benenson en los siguientes términos: "La aparición de casos de una enfermedad en evidente exceso de lo esperado" (1). En su diccionario de epidemiología (2), Last añadió a dicha definición el concepto de espacio - "en una comunidad" - y la amplió a otros acontecimientos relacionados con la salud, manteniendo la idea de "un evidente exceso". En ambas definiciones, está implícita una comparación entre el número de casos detectados y una cifra "normal de casos". Esa cifra "normal" depende obviamente de la población, del área geográfica y del período de tiempo que se considere. Este último punto es especialmente importante en aquellas enfermedades agudas en las cuales la estacionalidad desempeña un papel importante.

\footnotetext{
Las solicitudes de separatas deben pedirse a este autor a la siguiente dirección postal: Subsecretaría de Salud de Neuquén, Fotheringham 121, 8300 Neuquén, Argentina.
}

La palabra epidemia tiene un "aura pestilente" que explica el que muchos prefieran usar en su lugar el término "brote" para referirse al exceso de casos de una enfermedad o daño. Aunque al principio la definición de epidemia fue concebida para las enfermedades transmisibles, su uso es totalmente compatible con las no transmisibles. Así, por ejemplo, durante los fines de semana largos (con viernes o lunes festivos) se producen verdaderos "brotes" o epidemias de accidentes de tráfico. Por otra parte, Johan Giesecke destacó que en dicha definición no solo debe considerarse el número de casos (tiempo y lugar), puesto que una distribución poblacional particular (tiempo, lugar y persona) puede estar poniendo de manifiesto un número de casos mayor del esperado para ese grupo poblacional específico (mujeres, trabajadores de una industria en particular, personas con un modo de vida característico, etc.) (3).

Teniendo en cuenta lo expuesto, a la hora de realizar tareas de vigilancia epidemiológica en una población, será fundamental conocer cuán endémica es la enfermedad objetivo, es decir, cuál es la incidencia habitual de la enfermedad en dicha población o grupo de personas y en ese momento del año. Es en este punto donde se hace necesario preparar corredores o canales endémicos en los cuales la incidencia actual pueda ser representada gráficamente sobre la incidencia histórica y, de esta forma, detectar tempranamente cifras anormalmente altas (o bajas) de casos de la enfermedad en estudio.

En 1970, Héctor Boffi Borggero y Carlos Álvarez Herrera describieron varios métodos para elaborar corredores endémicos (4). Estos métodos consisten en calcular una medida central y un recorrido de fluctuación normal de la incidencia para cada uno de los meses, a partir de una serie de casos notificados en un período de 5 a 7 años. El más sencillo consistía en representar gráficamente el número máximo y mínimo de casos notificados cada mes, generando así una "banda 
endémica" con un área inferior de seguridad y una superior o epidémica. Un poco más complejo es el método de la mediana y los cuartiles, mediante el cual se generan cuatro zonas: una debajo del cuartil inferior o zona de éxito, una entre el cuartil inferior y la mediana o zona de seguridad, una entre la mediana y el cuartil superior o zona de alerta, y una por encima del cuartil superior o zona epidémica. Los modelos más complejos eran los de los mínimos cuadrados (que analizaban las tendencias lineales de cada año) y el de la media aritmética y desviaciones estándar, que precisaban para su aplicación de personal con considerables conocimientos en estadística. En estos últimos también se empleaban las cuatro zonas ya descritas (figura 1 ).

Todos estos métodos pueden considerarse modelos matemáticos más o menos complejos por medio de los cuales se pretende pronosticar, a partir de cinco o siete cifras, el número de casos que cabría esperar para ese período del año con un recorrido superior y uno inferior. Obviamente, cuanto mejor sea el modelo deseado, tanto mayor será el número de cálculos que deberán llevarse a cabo. Con el uso cada vez más frecuente de computadoras y programas estadísticos, que facilitan la realización de cálculos repetitivos y la representación gráfica de los resultados de los cálculos, estos modelos complejos pueden construirse sin la necesidad de disponer de personal especializado en estadística. Ello facilita la realización de corredores endémicos en niveles locales y regionales destinados a vigilar la incidencia de diferentes acontecimientos de salud. De esta forma, aumentará la sensibilidad de los sistemas de vigilancia para detectar cambios del número esperado de casos, que desde la perspectiva de los niveles centrales podrían verse diluidos.

\section{Factores que deben tenerse en cuenta antes de elaborar un corredor endémico}

La selección de la entidad, las poblaciones, la serie de años que van a incluirse y los intervalos de tiempo de-

\section{FIGURA 1. Esquema con las cuatro zonas de los corredores endémicos}

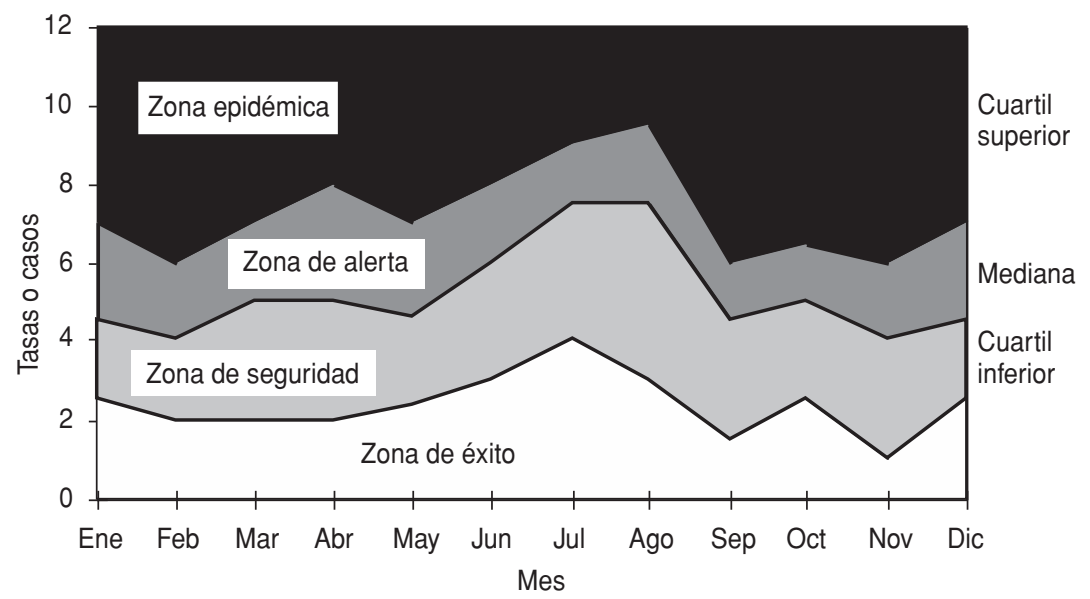

terminarán el grado de precisión de los corredores endémicos. En enfermedades de baja incidencia, en poblaciones pequeñas o con intervalos de tiempo cortos, el papel que desempeña el azar se hace más prominente. La consiguiente inestabilidad o dispersión de los casos notificados en los años previos condiciona notablemente la posibilidad de realizar predicciones, lo cual resulta en corredores de líneas dentadas con anchas áreas de seguridad y alarma. Es posible afirmar en esos casos que, a mayor nivel de desagregación de la información, menor será la precisión de la predicción. Otra posibilidad para enfermedades endémicas de baja incidencia es la realización de corredores acumulativos. Se trata de corredores endémicos en los cuales no se utiliza la incidencia semanal, sino que en su lugar el gráfico se construye a partir de la incidencia acumulada. En el presente trabajo, después de describir los pasos necesarios para realizar un corredor endémico con planillas de cálculos, se indicará cómo realizar un paso intermedio adicional que permitirá representar gráficamente corredores acumulativos.

Criterios de selección de entidades patológicas. Al seleccionar una enfermedad para realizar un corredor endémico, habrá que tener presente que se trate de una enfermedad endémica cuyo período de incubación sea breve y su evolución, aguda. No tendrá sentido realizar corredores endémicos para enfermedades de muy baja frecuencia en las cuales la sola presencia de uno o pocos casos debe alertar a los sistemas de vigilancia. De igual modo, en las enfermedades de evolución crónica, la acumulación de nuevos casos en un breve período de tiempo no tendrá, en general, mayor significación para la vigilancia epidemiológica $y$, seguramente, será la consecuencia de cambios o de la intensificación de los métodos diagnósticos (tamizaje) o simplemente del azar. Solo en situaciones muy excepcionales será la consecuencia de una verdadera epidemia, como lo fue el sarcoma de Kaposi durante el inicio de la epidemia del sida.

Agrupación de entidades patológicas. Cuando la incidencia lo permita, será útil realizar corredores independientes para cada enfermedad y sus subgrupos, por ejemplo, diarreas por grupos de edad o por agentes etiológicos. En el otro extremo, como es el caso de las poblaciones pequeñas, se podrían mantener agrupadas las entidades con formas de transmisión similares, vigilando no el comportamiento de un microorganismo o de una enfermedad, sino el de una vía de transmisión.

Poblaciones. Al igual que en el caso anterior, mantener la información y 
los corredores endémicos desagregados por pequeñas áreas geográficas favorecerá la posibilidad de detectar pequeños brotes locales que se diluirían en cifras acumuladas de zonas más amplias. No obstante, este nivel de desagregación deberá equilibrarse con el aumento de la imprecisión de los resultados. Cabe destacar aquí que, en ciertas circunstancias, es justamente la vigilancia en poblaciones más grandes y su consiguiente aumento de precisión lo que permite detectar un brote. Así, es posible que en varias áreas se genere un número de casos mayor que la media, pero sin ingresar en la zona de alarma, mientras que sobre el corredor endémico de la zona este "ligero" aumento en los niveles locales resulte en un ascenso "notable" en la zona.

Otro punto que ha de tenerse en consideración es el de los cambios que se observan en el número de habitantes de una población con el transcurso de los años. Por esta razón, no será adecuado analizar los datos como casos. Siempre que sea posible, se deberá disponer de cifras de población que permitan realizar corredores utilizando tasas de incidencia. Del mismo modo, si las enfermedades se consideran según ciertos grupos de edad, será preciso trabajar con tasas de incidencia específicas para esos grupos, al mismo tiempo que habrá de disponerse de la información de habitantes por grupos de edad y para cada año de las series incluidas.

Longitud de las series e intervalos de tiempo. Habitualmente, los corredores se construyen con series de casos de 5 a 7 años. Es lícito presuponer que, al aumentar el número de años, mejorará el modelo de predicción, pero deberá tenerse en cuenta que si se analizan series muy largas, es probable que tanto las condiciones que mantienen la endemia como los criterios diagnósticos y los mecanismos de notificación y registro hayan cambiado. Esto no es igual en todas las entidades, aunque, en líneas generales, si se considera una serie de 15 años, las condiciones de hace 15 años difícilmente podrán compararse con las actuales. Este tipo de cambio también deberá tenerse en cuenta aunque se considere un período de pocos años. Por ejemplo, si acaban de realizarse mejoras sustanciales en la red de cloacas de una localidad, la incidencia de enfermedades de transmisión fecal-oral debería ser menor que la "pronosticada" por el corredor endémico.

En cuanto a los intervalos de tiempo, siempre sería deseable realizar corredores por semanas epidemiológicas, pero ante incidencias bajas habrán de utilizarse períodos mayores, (2 ó 4 semanas), lo cual tenderá a estabilizar las fluctuaciones debidas al azar. La desventaja que supone utilizar períodos de 2 a 4 semanas es que se reduce la posibilidad de detectar tempranamente los brotes y que se retrasa la implantación de las consiguientes medidas de control.

\section{Selección del programa para elaborar el corredor}

Si bien existe la posibilidad de desarrollar programas específicos para realizar corredores endémicos, estos funcionarían como una "caja negra". Los datos serían introducidos en un extremo de la "caja" y los corredores "saldrían" por el otro extremo, distanciando a quien realiza la vigilancia epidemiológica de todo el proceso de su elaboración. Por esta razón, y considerando el amplio uso de las plantillas de cálculo y las facilidades que estos programas ofrecen para realizar cálculos y construir gráficos, este tipo de herramienta es la más adecuada para la tarea.

Las plantillas de cálculo a las que aquí se hará referencia son las siguientes: QPro versión 4.0 ó 5.0 y Microsoft Excel versión 5.0 y 97. Se sobrentiende que los usuarios han de tener cierta experiencia en la utilización de estos programas. $^{2}$

\footnotetext{
2 Los archivos para planillas Qpro y para Microsoft Excel con corredores semanal, cuatrisemanal y de incidencia acumulada vacíos y listos para incluir los casos de las series en el bloque 1 y otros archivos de ejemplos pueden solicitarse por correo electrónico a la siguiente dirección: Bortman@altavista.net
}

\section{Método de las medias geométricas de las tasas}

El método de la media geométrica de las tasas y sus intervalos de confianza es uno de los más sólidos desde el punto de vista estadístico. Como ya se ha mencionado, el uso de tasas en lugar de casos ajusta las distorsiones que generan los cambios en los tamaños de las poblaciones con el transcurso de los años.

\section{Media geométrica}

Teniendo en cuenta que las cinco o siete tasas con las que hay que calcular un valor central difícilmente tengan una distribución normal, el uso de la media aritmética no sería lo más adecuado. En cambio, la media geométrica es una medida de tendencia central apropiada para distribuciones de valores asimétricos o no normales y está especialmente indicada para distribuciones con valores aislados altos o muy altos. Esto último explica el hecho de que las posibles cifras muy elevadas que se detectan en alguna epidemia declarada durante la serie de tiempo incluida en la construcción del corredor se diluyan y no distorsionen la incidencia histórica. Para calcular la media geométrica $(\mu)$, se ha de realizar una transformación logarítmica de los valores mediante la siguiente formula.

$$
\mu=\log x
$$

donde $x$ es el valor inicial, y $\mu$, el valor transformado.

Esta transformación "estira" los valores bajos y "comprime" los altos. A continuación, todos los cálculos (de medias, desviaciones estándar e intervalos de confianza) se realizan con los valores transformados. Finalmente, los datos obtenidos se convierten en las unidades originales, calculando el antilogaritmo o, lo que es lo mismo, calculando $10^{\mu}\left(x=10^{\mu}\right)$. Otros tipos de logaritmos (por ejemplo, los de base n o naturales) pueden utilizarse con los mismos resultados:

$$
\mu=\ln (x) \text { y } x=\exp (\mu)
$$


En el cuadro 1 aparecen las tasas de sarampión del período comprendido entre 1983 y 1989 en la provincia del Neuquén, Argentina. En el mismo figuran la media aritmética y la geométrica de las tasas y sus respectivas desviaciones estándar. Como puede apreciarse, el "brote" de 1984 (tasa = 37,92/100 000) y la "epidemia" de 1989 $(136,43)$ afectaron mucho más a la media aritmética $(27,64)$ y a su desviación estándar $(49,7)$ que a la media geométrica $(7,1)$ y a su respectiva desviación estándar $(5,88)$.

Una limitación del cálculo de las medias geométricas es que los valores deben ser siempre mayores de 0 , ya que no es posible calcular el logaritmo de 0 ni de números negativos. Esto constituye una dificultad, pues es probable tener para algunos períodos tasas igual a 0. Para sortear ese escollo, Betty Kirkwood recomienda sumarles 1 a todos los valores originales, realizar todos los cálculos $\mathrm{y}$, finalmente, restar 1 a los valores finales (5).

\section{Recorridos superior e inferior}

Para calcular los recorridos superior e inferior, se puede usar la desviación estándar de las tasas o el intervalo de confianza de la media de las tasas. La desviación estándar tiene en cuenta la dispersión de los datos que dieron origen a la media (los de la serie). Así, al considerar la media \pm 1 desviación estándar se estarán incluyendo 68,26\% de los valores que dieron origen a la media. Si se consideran 2 desviaciones estándar, se estarán incluyendo 95,45\% de esos valores. Por lo tanto, al utilizar las desviaciones estándar en la construcción de los recorridos superior e inferior se estarán comparando las tasas del corriente año con las de los años anteriores, no con su media. Una tasa que supere el recorrido superior e ingrese en la zona epidémica será entonces similar o mayor a los valores más altos de la serie de los últimos años.

El concepto de intervalo de confianza (IC) de la media es distinto, ya que comprende el recorrido de valores en el cual se encontraría, con un determinado nivel de confianza, la "verdadera media del universo" bajo el supuesto de que las 5 ó 7 tasas usadas para el cálculo constituyen una muestra del mismo. Un IC95\% será, por lo tanto, el conjunto de valores de la media del universo compatibles con los datos observados con una confianza del $95 \%$ o, en otras palabras, el conjunto de valores compatibles con los datos observados entre los cuales cabe esperar, con una confianza de $95 \%$, que se encuentre la media verdadera del universo. Al usar el intervalo de confianza en la construcción de los corredores, se estarán comparando las tasas del año en curso con la "verdadera media" de las tasas del período anterior. A su vez, con una confianza de $95 \%$, esperaremos que la tasa que supere el límite superior del corredor e ingrese a la zona epidémica sea mayor que la "verdadera media histórica". El uso de intervalos de confianza dará

CUADRO 1. Tasas de sarampión (por 100000 habitantes), media aritmética, media geométrica y desviación estándar (DE). Provincia del Neuquén, Argentina, 1983-1989

\begin{tabular}{lrcc}
\hline Año & Tasa & $\begin{array}{c}\text { Escala } \\
\text { logarítmica } \\
\text { (tasa) }\end{array}$ & $\begin{array}{c}\text { Transformados } \\
\text { a unidades } \\
\text { originales }\end{array}$ \\
\hline 1983 & 2,13 & 0,76 & - \\
1984 & 37,92 & 3,64 & - \\
1985 & 4,84 & 1,58 & - \\
1986 & 1,54 & 0,43 & - \\
1987 & 1,17 & 0,16 & - \\
1988 & 9,46 & 2,25 & - \\
1989 & 136,43 & 4,92 & 7,10 \\
Media & 27,64 & 1,96 & 5,88 \\
DE & 49,70 & 1,77 & - \\
\hline
\end{tabular}

lugar a corredores con zonas de seguridad y alerta más estrechas y, por consiguiente, más sensibles a los cambios de la incidencia que los corredores construidos sobre la base de desviaciones estándar.

Si bien las plantillas de cálculo incorporan las fórmulas de la desviación estándar como funciones, no ocurre lo mismo para el cálculo del intervalo de confianza de la media. Por esta razón, será necesario escribir estás fórmulas en las celdas correspondientes.

$$
\mathrm{IC} 95 \%=\text { media } \pm t D E / \sqrt{n}
$$

donde $n$ es el número de años considerados en el cálculo, $D E$, la desviación estándar y $t$, un valor que se obtiene de la tabla de distribución de $t$ y cuyos valores varían en función de $n$.

Valores $t$ para IC95\% según los años incluidos en las series

$\begin{array}{cc}\text { Años (No.) } & t \\ 3 & 4,30 \\ 4 & 3,18 \\ 5 & 2,78 \\ 6 & 2,57 \\ 7 & 2,45 \\ 8 & 2,36 \\ 9 & 2,31 \\ 10 & 2,26 \\ 11 & 2,23 \\ 12 & 2,20\end{array}$

Por ejemplo, para calcular el IC95\% de la media geométrica de las tasas de incidencia de sarampión del período de 1983 a 1989, se deberá realizar el siguiente cálculo:

$$
\begin{gathered}
\mathrm{IC} 95 \%=1,96 \div 2,45 \times 1,77 / \sqrt{7}=1,96 \\
\pm 1,639=0,32 \text { a } 3,6
\end{gathered}
$$

Donde 1,96 y 1,77 son, respectivamente, la media y la desviación estándar en escala logarítmica; 7, la $n$ (7 años) y 2,45 , el valor de $t$ para $n=7$. Al convertir los resultados a la escala original se obtiene:

\section{Media geométrica de la tasa de incidencia $=7,10$ por 100000}

(IC95\%: 1,38 a 36,6) 
Nótese que los límites superior e inferior de los intervalos de confianza no son simétricos; sin embargo, la razón entre el límite superior y la media y entre la media y el límite inferior sí lo es:

$$
(36,6 / 7,10=7,10 / 1,38)
$$

\section{Elaboración en etapas}

Para elaborar el corredor endémico en una plantilla de cálculo, será necesario construir una serie de tablas y realizar cálculos en varias etapas, tal como se detalla a continuación.

Primera etapa: ingreso de los datos. El primer paso consiste en construir un bloque en el cual se ingresarán los casos detectados por años y períodos. Por ejemplo, el bloque 1 [A1 a BB10] con 52 semanas en las columnas y los años en las filas se ha preparado para ingresar los casos de 1990 a 1998. Nótese que se ha preparado la última columna (BB) para colocar en ella las poblaciones de cada año.

Segunda etapa: cálculo de las tasas. En el segundo paso se calculan las tasas de incidencia semanal y se suma 1 a cada una de ellas, con objeto de permitir calcular la media geométrica en caso de existir tasas $=0$. Para ello, se preparará un bloque similar debajo del anterior (bloque 2 [A15 a BA24]) y en cada celda del bloque se incluirá la fórmula correspondiente. La forma más rápida de hacerlo es incluir la formula en la casilla B16 y copiarla en las restantes casillas. La formula de la casilla B16 será:

$$
((+\mathrm{B} 2 / \$ \mathrm{BB} 2 * 100000)+1)
$$

donde B2 es el numero de casos de la semana 1 del año 1990, y BB2, la población en 1990. El signo \$ que precede a BB permite que, al copiar esta fórmula en otras casillas, se continúe utilizando la columna BB para obtener las poblaciones.

Bloque 1: casos por semana y años, y población por años

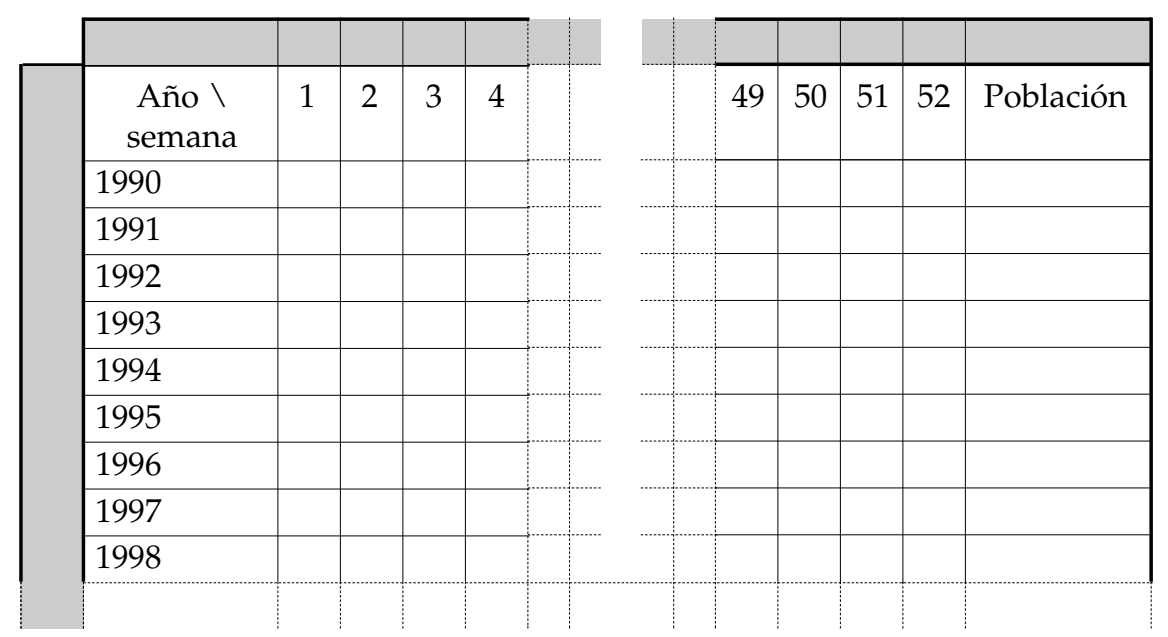

Bloque 2: cálculo de tasas de incidencia semanal más 1

D19: $((+\mathrm{D} 5$ / \$BB5 * 100000$)+1)$

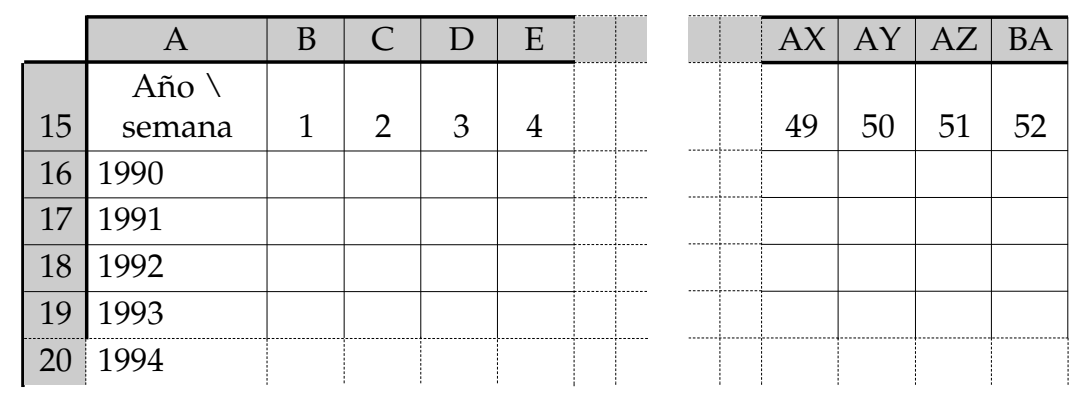

\section{Bloque 3: transformación logarítmica de las tasas}

\begin{tabular}{|c|c|c|c|c|c|c|c|c|c|}
\hline & A & B & $\mathrm{C}$ & D & $\mathrm{E}$ & $\mathrm{AX}$ & AY & $\mathrm{AZ}$ & BA \\
\hline 27 & $\begin{array}{c}\text { Año } \backslash \\
\text { semana }\end{array}$ & 1 & 2 & 3 & 4 & 49 & 50 & 51 & 52 \\
\hline 28 & 1990 & & & & & & & & \\
\hline 29 & 1991 & & & & & & & & \\
\hline 30 & 1992 & & & & & & & & \\
\hline 31 & 1993 & & & & & & & & \\
\hline 32 & 1994 & & & & & & & & \\
\hline
\end{tabular}

D19:@ln(D19))

Después de copiar las fórmulas, habrá que verificar este procedimiento y confirmar que las fórmulas se hayan copiado adecuadamente. Nótese, por ejemplo, que la casilla D19 contiene la fórmula $((+\mathrm{D} 5$ / \$BB5 *100 000) $+1)$.
Tercera etapa: transformación logarítmica de las tasas. Para transformar las tasas a la escala logarítmica, se utilizará un tercer bloque similar al anterior (bloque 3) y se usará la función ln $(\mathrm{x})$, donde $\mathrm{x}$ es la casilla correspondiente al bloque anterior. 
Cuarta etapa: cálculo de las medias, desviaciones estándar e intervalos de confianza de $\mathbf{9 5 \%}$ en escala logarítmica. Para calcular las medias, las desviaciones estándar y los IC95\% (bloque 4) serán necesarias otras cuatro filas. Dado que hay algunas diferencias en los nombres de las funciones entre QPro y Excel, las fórmulas empleadas en uno $u$ otro programa serán ligeramente distintas. En los ejemplos se utilizarán los datos entre 1990 y 1996 (filas 28 a 34); de ahí que en los cálculos de los intervalos de confianza $n=7$ y $t=2,45$.

Quinta etapa: transformación a unidades originales restando 1 , y conversión de tasas a casos esperados. El último paso consiste en convertir los valores obtenidos a su escala original, restarles 1 para restablecer el corrimiento de las tasas introducido al sumarle 1 en la segunda etapa, y convertir las tasas a casos esperados para facilitar la utilización del corredor. El bloque 5 muestra las fórmulas necesarias para realizarlo. La fila 45 contendrá la media geométrica y las filas 46 y 47, los límites superior e inferior de los intervalos de confianza del $95 \%$. En las filas 48 a 50 las tasas se convierten a casos esperados, teniendo en cuenta la población del año 1997 (celda BB9).

Sexta etapa: gráfico. Por último, con la media geométrica y su intervalo de confianza expresados en casos para la población del año en curso se estará en condiciones de construir un gráfico de áreas. Sobre este gráfico se deberán representar gráficamente a mano, semana a semana, el número de casos notificados como parte de la actividad de vigilancia.

Para facilitar la "superposición" de las áreas del gráfico, será conveniente construir otro bloque a fin de calcular, por un lado, la diferencia entre el límite inferior del intervalo de confianza y la media geométrica, $y$, por otro, la diferencia entre esta última y el límite superior de dicho intervalo (bloque 6).
Bloque 4: cálculo logarítmico (ln) de medias, desviaciones estándar e IC95\% en QPro y Microsoft Excel

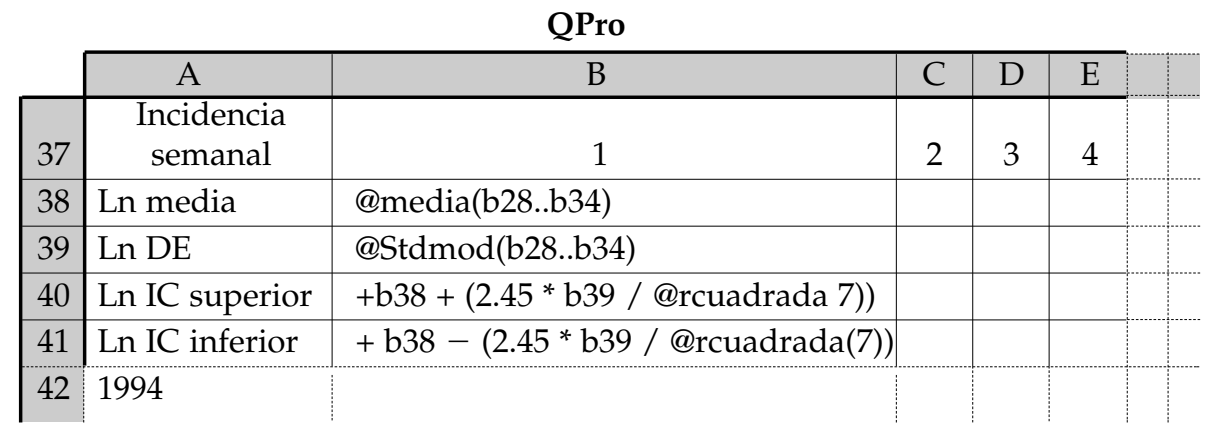

\section{Microsoft Excel}

\begin{tabular}{|c|c|c|c|c|c|}
\hline & $\mathrm{A}$ & $\bar{B}$ & $\mathrm{C}$ & $\mathrm{D}$ & $\bar{E}$ \\
\hline 37 & $\begin{array}{c}\text { Incidencia } \\
\text { semanal }\end{array}$ & 1 & 2 & 3 & 4 \\
\hline 38 & Ln media & $=$ media $(\mathrm{b} 28: \mathrm{b} 34)$ & & & \\
\hline 39 & Ln DE & $=\mathrm{DE}(\mathrm{b} 28: \mathrm{b} 34)$ & & & \\
\hline 40 & Ln IC inferior & $=$ b38 $-(2.45 *$ b39 / raíz $(7))$ & & & \\
\hline 41 & Ln IC superior & $=$ b38 $+(2.45 *$ b39 / raíz $(7))$ & & & \\
\hline 42 & & & & & \\
\hline
\end{tabular}

Bloque 5: transformación a unidades originales menos 1, y cálculo de casos

\begin{tabular}{|c|l|l|l|l|l|l|}
\cline { 2 - 7 } \multicolumn{1}{c|}{} & \multicolumn{1}{c|}{ A } & B & C & D & E & \\
\hline 44 & \multicolumn{1}{c|}{$\begin{array}{l}\text { Incidencia } \\
\text { semanal }\end{array}$} & 1 & 2 & 3 & 4 & \\
\hline 45 & IC inferior (tasa) & $@ \exp (\mathrm{b} 40)-1$ & & & & \\
\hline 46 & Media (tasa) & $@ \exp (\mathrm{b} 38)-1$ & & & & \\
\hline 47 & IC superior (tasa) & $@ \exp (\mathrm{b} 41)-1$ & & & & \\
\hline 48 & IC inferior (casos) & $+\mathrm{b} 45^{*} \$ \mathrm{bb} \$ 9 / 100000$ & & & & \\
\hline 49 & Media(casos) & $+\mathrm{b} 46^{*} \$ \mathrm{bb} \$ 9 / 100000$ & & & & \\
\hline 50 & IC superior (casos) & $+\mathrm{b} 47^{*} \$ \mathrm{bb} \$ 9 / 100000$ & & & & \\
\hline 51 & & & & & &
\end{tabular}

\section{Bloque 6: cálculo de las diferencias}

\begin{tabular}{|l|l|l|l|l|l|l|}
\cline { 2 - 7 } \multicolumn{1}{c|}{} & \multicolumn{1}{c|}{ A } & B & C & D & E \\
\hline 52 & IC inferior (casos) & $+\mathrm{b} 48$ & & & & \\
\hline 53 & Media-IC inferior & $@ \exp (\mathrm{b38)}-1$ & & & & \\
\hline 54 & IC superior-media & $@ \exp (\mathrm{b} 41)-1$ & & & & \\
\hline 55 & & & &
\end{tabular}




\section{Gráfico con QPro}

Desde menú-gráficos se creará un gráfico de áreas, se definirán como series 1, 2 y 3 las celdas A52..BA52, A53, BA53 y A54, BA54, respectivamente, y, pulsando $\langle\mathrm{F} 10\rangle$, podrá visualizarse. Posteriormente, se colocarán los títulos adecuados y ya estará listo para imprimirse. También se podrán realizar cambios en los colores, tramas y en otros detalles de la presentación gráfica.

\section{Gráfico con Microsoft Excel}

Se marca el bloque (A52..BA54) y, desde insertar-gráfico-en esta hoja, se ingresa al asistente para gráficos. Se selecciona un gráfico de áreas tipo 1 y se procede con el asistente hasta finalizar. Al igual que con QPro, y antes de imprimir, posteriormente se podrán añadir títulos, modificar los colores o tramas de las series, etc. (figura 2).

\section{Corredor endémico acumulativo}

Tal como se ha comentado, una alternativa para realizar corredores endémicos para enfermedades endémicas pero de baja incidencia es utilizar la incidencia acumulada en lugar de la incidencia semanal. De este modo, se evitará que el corredor presente una imagen muy dentada. Las figuras 3 y 4 muestran, respectivamente, el corredor endémico y el corredor endémico acumulativo para meningitis bacteriana en la provincia del Neuquén correspondientes al período comprendido entre 1990 y 1996. En ambos corredores también se representó gráficamente la incidencia del año 1997 hasta la semana 31 como una línea negra. Como puede verse, la vigilancia de la incidencia actual se simplifica en la figura 4 de incidencia acumulada.

Para realizar corredores con incidencia acumulada, bastará introducir la incidencia acumulada semanal en lugar de la incidencia semanal o incluir un bloque que acumule la incidencia a partir de los datos de incidencia semanal.
FIGURA 2. Corredor endémico de diarreas en mayores de 5 años. Rincón de los Sauces, Argentina, 1990-1996

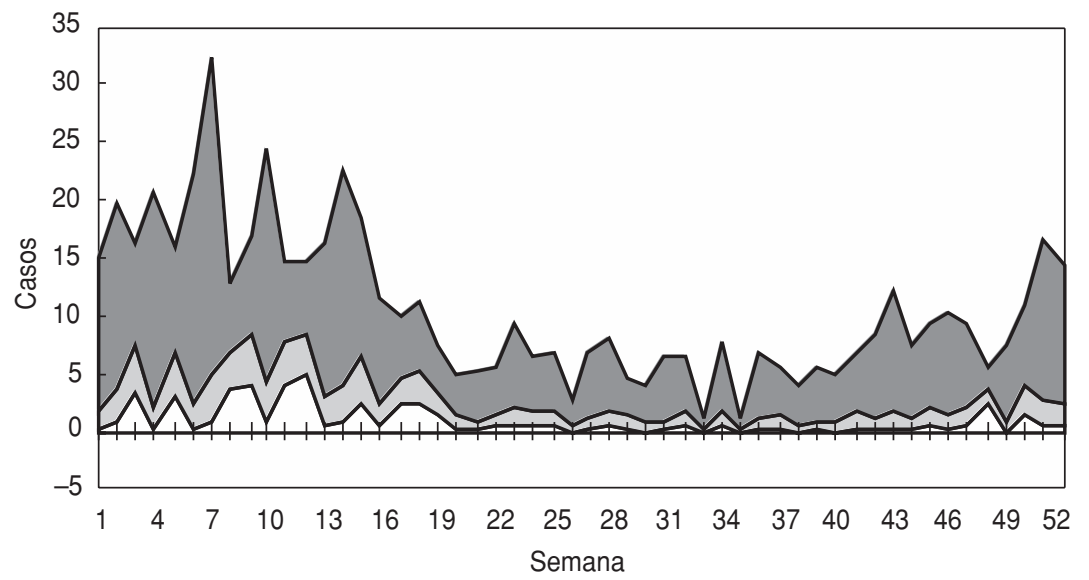

FIGURA 3. Corredor endémico de meningitis bacteriana. Provincia del Neuquén, Argentina, 1990-1996 e incidencia (semana 31), 1997

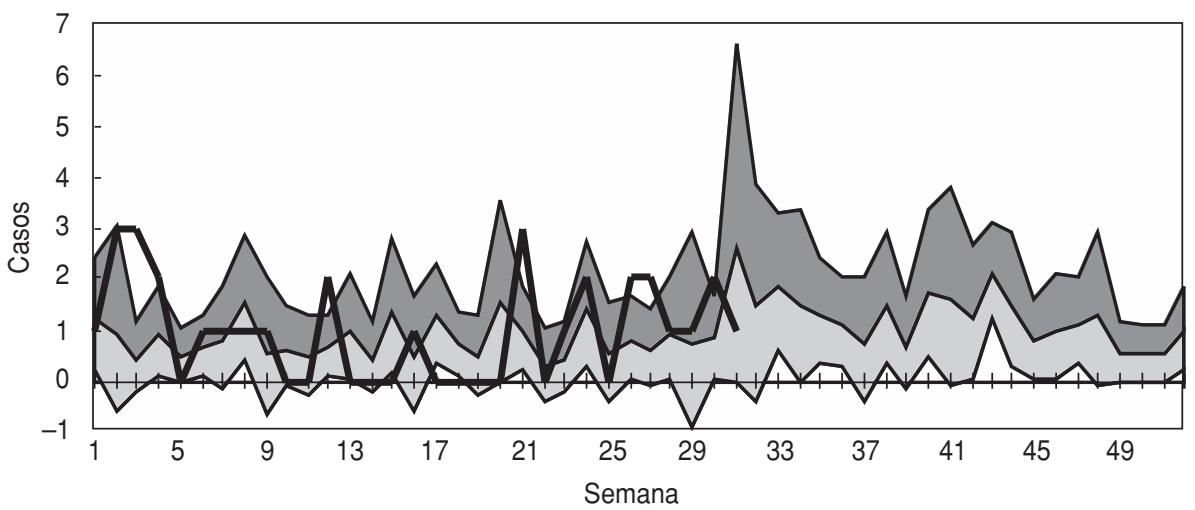

FIGURA 4. Corredor endémico acumulado de meningitis bacteriana. Provincia del Neuquén, Argentina, 1990-1996 e incidencia (semana 31) 1997

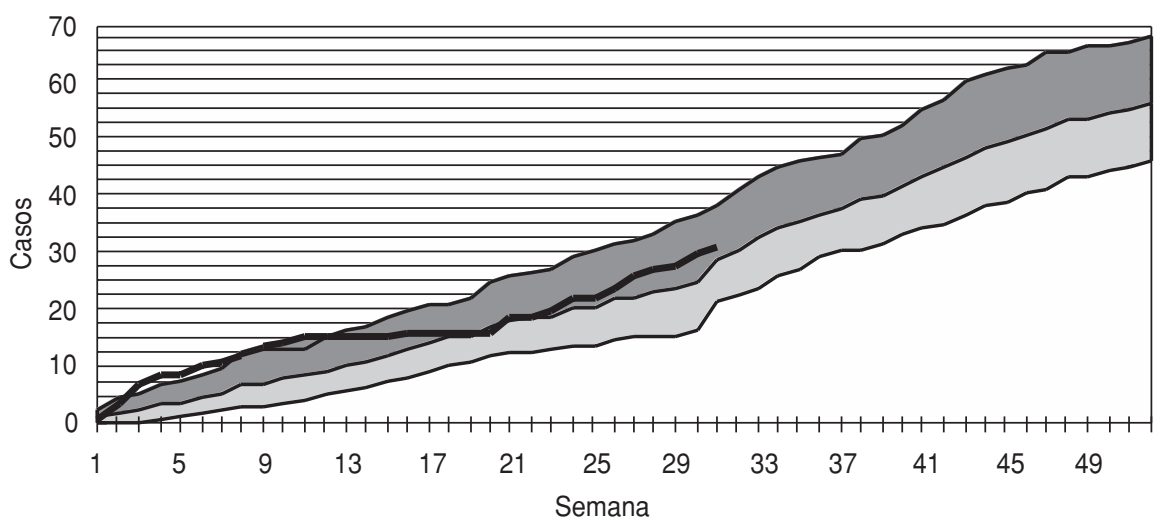




\section{REFERENCIAS}

1. Benenson AS. Control of communicable diseases in man. 15a ed. Washington, D.C.: American Public Health Association; 1990.

2. Last JM. A dictionary of epidemiology. 2a ed. Oxford: Oxford University Press; 1995.

3. Giesecke J. Modern infectious disease epidemiology. New York: Oxford University Press; 1994.
4. Boffi H, Álvarez-Herrera C. Contribución al estudio de las enfermedades transmisibles mediante el uso de algunos métodos estadísticos. Salud Argentina 1970;1:13-97.

5. Kirkwood B. Essentials of medical statistics. Oxford: Blackwell Science; 1988.
Manuscrito recibido el 3 de septiembre de 1997 y aceptado para publicación en versión revisada el 26 de agosto de 1998.

ABSTRACT Epidemics or disease outbreaks can be defined as an excess in the number of cases of a given health problem, in a particular population, period, and place. However, to determine what constitutes an excess implies knowing what is normal or to be expected. Establishing endemic levels or ranges makes it possible to determine figures for the number of expected cases and to show in graphic form the appearance of a higher number of cases. In this work a new methodology is described to develop these endemic ranges, in which the Quattro Pro (Qpro) and Excel software programs are used to calculate the geometric mean of historical rates and their confidence intervals. Also presented is a procedure for cumulative endemic ranges that facilitates surveillance of endemic events with a low incidence. 